\title{
Solution of the Quantum Initial Value Problem with Transparent Boundary Conditions
}

\author{
A. Puga and B.N.Miller \\ Department of Physics and Astronomy, Texas \\ Christian University, Fort Worth, Texas 76129, USA
}

\begin{abstract}
Physicists have used billiards to understand and explore both classical and quantum chaos. Recently, in 2001, a group at the University of Texas introduced an experimental set up for modeling the wedge billiard geometry called optical billiard in two dimensions. It is worth mentioning that this experiment is more closely related with classical rather than quantum chaos. The motivation for the present work was born from the idea of laying the foundations of a quantum treatment for optical billiards, named "The Escape Problem", by presenting the concept of a Transparent Boundary Condition. We consider a "gas of particles" initially confined to a one dimensional box of length $L$, that are permitted to escape. We find the solution of a Quantum Initial Value Problem using a numerical method developed and entirely checked with an exact, analytic method. The numerical method introduces a novel way to solve a Diffusion Type Equation by implementing Discrete Transparent Boundary Conditions recently developed by mathematicians.
\end{abstract}

PACS numbers: 


\section{INTRODUCTION}

The seminal model for understanding both classical and quantum chaos is the billiard. Depending on different considerations, such as shape and topology, a billiard model can present either stable or chaotic behavior. The most celebrated versions of classical billiards in two dimensions, in which chaos has been observed, are the Sinai billiard $\mathbb{1}$, Bunimovich stadium $^{2}$, and Polygonal billiards ${ }^{3}$. In all of these examples, the shape of the boundaries plays a crucial role. In recent years, physicists have detected chaotic behavior at the nano scale ${ }^{4}$ making an important impact in technology and, once again, billiards have helped to model quantum devices ${ }^{5}$ such as nanotubes, quantum dots, etc. which are governed by quantum theory.

A novel model of billiard is the one introduced in 1986 by Lehtihet and Miller ${ }^{\underline{6}}$ known as the "Wedge Billiard." The model consists of a symmetrically inclined wedge of angle $2 \theta$ with respect to the direction of a constant gravitational field $\mathbf{g}$ in which the particle is confined. They found surprising properties when the parameter $\theta$ is changed. Later, in 2001, Valery Milner ${ }^{7}$, working in Mark Raizen's laboratory at the University of Texas, introduced an experiment referencing the Wedge Billiard model geometry and confirming the properties mentioned above. This first experiment of billiards was named "Optical Billiards." It is worth mentioning that this experiment is more closely related to classical rather than "quantum" chaos. While very low by normal standards, the temperature they employed is not low enough to easily exhibit any quantum effects. Consequently, a classical theoretical model was adequate to obtain a good point of comparison.

A key element in the experiment is that, by removing a small segment of the boundary, an escape route is provided at the billiard vertex. As time progresses, the atoms, moving under the influence of gravity, will eventually exit the billiard. A measure of the influence of "chaotic" orbits is provided by the mean lifetime that the atoms remain in the billiard. This scenario is mimicked in this work by setting up the Escape Problem (EP). Currently experimentalists are starting to probe the quantum regime.

The idea of the work presented here springs from laying the foundation of a quantum treatment for optical billiards. However, the quantum mechanical problem is much more difficult since the system is two-dimensional (equivalent to four dimensions in the phase space), and not integrable. Thus analytical methods are not available, and a viable numerical 
method for solving the Schrödinger equation is required. Since it is extremely difficult to develop a numerical method for a two dimensional integro-differential equation with nonlocal transparent boundary conditions and skew boundaries, this work will develop an essential numerical method for obtaining the solution of the non-relativistic one-dimensional Schrödinger equation.

The most demanding part of this work is the implementation of Transparent Boundary Conditions $(T B C s) \stackrel{8.9}{.}$. The $T B C s$, recently developed by mathematicians, arise in the necessity to deal numerically with the natural infinite domain of the wave function. That is, due to the limitation of the computer core size, the infinite wave solution of the Schrödinger equation has to be solved in a finite domain by imposing artificial boundary conditions. The criterion of transparency is that the incident wave at the boundary has the smallest reflection coefficient possible. If the solution with these artificial conditions agrees with the infinite solution, the artificial boundary conditions are said to be transparent. From the several available approaches to derive $T B C s$, this work is based on the excellent treatment carried out by Anton Arnold $\underline{\underline{8}}$ and his student Mathias Earhardt $\underline{9} \cdot 10$ that concerns the transport of a quantum particle that enters one side of a finite domain and exits from the opposite side.

In the following, in the next section, we first set up the "Escape Problem, i.e. the escape of a particle from a finite region, as a Quantum Initial Value Problem (QIVP). We then develop two different approaches for solving the EP. The first is the analytic method that provides the certainty of the result. The second is the numerical method, where an algorithm will be meticulously presented by introducing Discrete Transparent Boundary Conditions $(D T B C)^{10}$, based on the implicit Crank-Nicholson method. Subsequently, the consistency of both methods is confirmed in section III by comparing the solutions for the real and imaginary parts of the wave function at different times, showing an excellent agreement. In the last section, conclusions are drawn from the work that has been accomplished, stressing the valuable physical information provided by the wave function as well as the numerical importance of the method developed in this work. 


\section{THEORY}

\section{A. DESCRIPTION OF THE SYSTEM.}

The system representing the one-dimensional Escape Problem (EP) is a group of particles restricted inside a region of size $L$ delimited by boundaries and free of external influences where, abruptly, one of the boundaries becomes transparent, allowing particles to escape. The group of particles is a dilute gas rarefied enough to be considered as a "Knudsen Gas"11 whose density is so small that only the interactions with the boundaries are relevant. From the perspective of quantum mechanics, the EP is a QIVP. Thus, the intention is to solve the 1-D Schrödinger equation from a given initial condition. The EP is similar to a diffusion type problem; it has the same structure as propagation problems in which a partial differential equation is solved with the aid of a known initial value. The evolution of the gas inside the region $0<x<L$ will be determined by the wavefunction at the initial time. To demonstrate the approach, here the initial wavefunction is chosen to be the ground state of an Infinite Well Potential of length $L$. Therefore, the initial value or the initial condition inside of the box is

$$
\Psi_{I}(x, 0)=\sin (k x)
$$

with $k=\frac{\pi}{L}$. Of course, outside of the box the value of the initial wave function vanishes. The evolution of the EP is represented in the figure 1.

\section{B. ANALYTICAL METHOD.}

The non-relativistic, time dependent, one-dimensional Schrödinger equation is

$$
\frac{\partial \Psi(x, t)}{\partial t}=i \frac{\hbar}{2 m} \frac{\partial^{2} \Psi(x, t)}{\partial x^{2}}
$$

with the initial condition:

$$
\left.\Psi(x, t)\right|_{t=0}=\Psi_{I}(x, 0) .
$$

The Laplace Transform Method is one of the many appropriate techniques to solve differential equations. The first task is to determine the Laplace transform of the wave function and its time derivative. For this particular example the definition of the Laplace transform 


$$
\phi(x, s)=\int_{0}^{\infty} e^{-s t} \Psi(x, t) d t
$$

or,

$$
\phi(x, s)=\mathcal{L}\{\Psi(x, t)\}
$$

By integrating by parts,

$$
\mathcal{L}\left\{\frac{d \Psi(x, t)}{d t}\right\}=s \phi(x, s)-\Psi_{I}(x, 0) .
$$

After using equations (4) and (5), the Schrödinger equation (2), after some algebraic re-arrangements, becomes:

$$
\frac{\partial^{2} \phi(x, s)}{\partial x^{2}}+i \alpha s \phi(x, s)=i \alpha \Psi_{I}(x, 0)
$$

with

$$
\alpha=\frac{2 m}{\hbar}
$$

A Green's Function can be used to solve the inhomogeneous differential equations (66). The following notation and definitions are necessary before using the Green's Function.

The Laplace transform of the Green's function is denoted as:

$$
\mathcal{L}\left\{G\left(x, x^{\prime}, t\right)\right\}=g\left(x, x^{\prime}, s\right)
$$

where $G\left(x, x^{\prime}, t\right)$ is the solution in the non-transformed space. The Green's function for equation (6) satisfies

$$
\frac{d^{2} g\left(x, x^{\prime}, s\right)}{d x^{2}}+i \alpha s g\left(x, x^{\prime}, s\right)=\delta\left(x-x^{\prime}\right)
$$

and its solution is given by

$$
\phi(x, s)=i \alpha \int_{0}^{\infty} d x^{\prime} g\left(x, x^{\prime}, s\right) \Psi_{I}\left(x^{\prime}, 0\right) .
$$


The complete time dependent solution of the initial value problem $\Psi(x, t)$ is obtained by inverting the Laplace transform:

$$
\Psi(x, t)=i \alpha \int_{0}^{\infty} d x^{\prime} G\left(x, x^{\prime}, t\right) \Psi_{I}\left(x^{\prime}, 0\right)
$$

The remaining procedure consists in constructing the Green's function $g\left(x, x^{\prime}, s\right)$ for our particular problem, establishing $G\left(x, x^{\prime}, t\right)$, and finally substituting it into equation (11).

In order to construct the Green's Function $g\left(x, x^{\prime}, s\right)$, beginning from equation (9) for $x \neq x^{\prime}$ and assuming $\operatorname{Re} P>0$, the form of the postulated solution is $g=e^{ \pm P x}$. Thus,

$$
P=\sqrt{\frac{\alpha s}{2}}(1-i)
$$

For $x<x^{\prime}$

$$
\begin{array}{rll}
g_{1} & = & B e^{P x}+C e^{-P x} \\
x=0 & & g_{1}(0)=0 \\
B+C=0 & \\
g_{1}= & B\left(e^{P x}-e^{-P x}\right)
\end{array}
$$

And for $x>x^{\prime}$

$$
g_{2}=A e^{-P x}
$$

The following two conditions give $A$ and $B$. For continuity $g_{1}=g_{2}$, and for the discontinuity of the derivative $\left.\frac{d g_{2}}{d x}\right|_{x^{\prime}}-\left.\frac{d g_{1}}{d x}\right|_{x^{\prime}}=1$.

These two conditions lead to the value of:

$$
B=-\frac{1}{2 P} e^{-P x^{\prime}}
$$

Using this value of $B$, the value for $A$ is derived as

$$
A=\frac{1}{2 P}\left(e^{P x^{\prime}}-e^{-P x^{\prime}}\right) .
$$


The equation (14) and the equation (15) can be rewritten to obtain:

$$
\begin{aligned}
\text { for } x & <x^{\prime} \\
g_{1} & =-\frac{1}{2 P} e^{-P x^{\prime}}\left(e^{P x}-e^{-P x}\right) \\
\text { for } x & >x^{\prime} \\
g_{2} & =-\frac{1}{2 P}\left(e^{P x^{\prime}}-e^{-P x^{\prime}}\right) e^{-P x} .
\end{aligned}
$$

Alternatively,

$$
\begin{aligned}
g_{1} & =g_{1 a}+g_{1 b} \\
g_{1 a} & =-\frac{1}{2 P} e^{-P\left(x^{\prime}-x\right)} \\
g_{1 b} & =\frac{1}{2 P} e^{-P\left(x+x^{\prime}\right)}
\end{aligned}
$$

and,

$$
\begin{aligned}
g_{2} & =g_{2 a}+g_{2 b} \\
g_{2 a} & =-\frac{1}{2 P} e^{-P\left(x-x^{\prime}\right)} \\
g_{2 b} & =\frac{1}{2 P} e^{-P\left(x+x^{\prime}\right)} .
\end{aligned}
$$

In general, the inversion of the Laplace transform of any of the $g$ functions has to be done via contour integration in the complex $s$ plane. In addition, extensive mathematical tables are also available with many worked out examples $\frac{12}{}$. This section shows only one of the $g$ functions, $g_{1 a}$, as an illustration of the use of these tables (the rest of functions $g_{1 b}, g_{2 a}, g_{2 b}$ are easy to obtain due to their similarity). The following expressions can be found 12

$$
\begin{aligned}
f(s) & F(t) \\
\frac{1}{\sqrt{s}} e^{-k \sqrt{s}} & \frac{1}{\sqrt{\pi t}} e^{-\frac{k^{2}}{4 t}}
\end{aligned}
$$


for $k>0$. Then, using (7) and (12) it is easy to see that

$$
g_{1 a}=\frac{e^{-(1-i) \sqrt{\frac{m s}{\hbar}}\left(x^{\prime}-x\right)}}{2(1-i) \sqrt{\frac{m s}{\hbar}}}
$$

and its corresponding inverse Laplace transform

$$
G_{1 b}=\frac{e^{-\frac{1}{4 t}\left[\frac{m}{h}(1-i)^{2}\left(x^{\prime}+x\right)\right]}}{2(1-i) \sqrt{\pi t \frac{m}{\hbar}}}
$$

Similarly, it is easy to obtain

$$
\begin{aligned}
G_{2 a} & =-\frac{e^{-\frac{1}{4 t}\left[\frac{m}{h}(1-i)^{2}\left(x^{\prime}-x\right)\right]}}{2(1-i) \sqrt{\pi t \frac{m}{\hbar}}} \\
G_{2 b} & =\frac{e^{-\frac{1}{4 t}\left[\frac{m}{h}(1-i)^{2}\left(x^{\prime}+x\right)\right]}}{2(1-i) \sqrt{\pi t \frac{m}{\hbar}}} .
\end{aligned}
$$

Defining the following constants $C \equiv \frac{1}{2(1-i) \sqrt{\frac{m \pi}{\hbar}}}$, and $a \equiv \frac{m}{2 \hbar}$ the total Green's function is found to be:

$$
G\left(x . x^{\prime}, t\right)=\frac{C}{\sqrt{t}}\left[-e^{\frac{i a}{t}\left(x^{\prime}-x\right)^{2}}+e^{\frac{i a}{t}\left(x^{\prime}+x\right)^{2}}\right] .
$$

Once the Green's function is constructed by the above procedure, in order to obtain the wave function it is necessary to substitute equation (28) into the equation (11) resulting in the complete solution:

$$
\Psi(x, t)=i \frac{\alpha C}{\sqrt{t}} \int_{0}^{\infty} d x^{\prime}\left(-e^{\frac{i a}{t}\left(x^{\prime}-x\right)^{2}}+e^{\frac{i a}{t}\left(x^{\prime}+x\right)^{2}}\right) \Psi_{I}\left(x^{\prime}, 0\right)
$$

Mathematica was employed for these computations and for plotting the results.

\section{NUMERICAL METHOD.}

As mentioned earlier, the QIVP can be treated as a propagation problem. These are initial-value problems governed by a parabolic Partial Differential Equation $(P D E)$ of first order in the time. A familiar representation of a parabolic PDE in one dimension is the Diffusion Equation,

$$
f_{t}=\alpha f_{x x}
$$


Subscripts $t$ and $x x$ represent the first time derivative and second position derivative of the function $f$. The factor $\alpha$ in the equation is called the diffusivity which is defined by the system under investigation. The Schrödinger equation (in dimensionless units) using the same notation as in equation (29) becomes

$$
i \Psi_{t}=-\frac{1}{2} \Psi_{x x}
$$

Except for the imaginary number $i$, the equation (30) is identical to (29). Therefore, it is mathematically correct to proceed to solve (30) with the complex extension of the same tools used in the numerical method for the Diffusion Equation. Numerical methods $\frac{13}{3}$ solve the $P D E$ by transforming the integral problem into an algebraic one that is computationally accessible. The Crank-Nicholson method is the preferred numerical algorithm used to solve the Schrödinger equation as a diffusion type equation. It is an implicit algorithm valid through second order in both space and time coordinates, so it is very stable ${ }^{13}$.

To make that equation equivalent to the escape problem, it is necessary to employ the mathematical concepts of TBCs. The discretized domain of the solution is constructed using the following finite difference grids

$$
\begin{aligned}
& x_{i}=(i-1) \triangle x \\
& t^{n}=n \triangle t(\triangle t \text { constant })
\end{aligned}
$$

with $i=0,1,2, \ldots, i \max$ and $n=1,2, \ldots, n \max$. The domain $D(x, t)$ is from 0 to $L$ on the $x$-axis and the solution is marching in a positive time direction on the $y-$ axis. We introduce the following notation in the context of the finite difference method grid point $(i, t) \rightarrow\left(x_{i}, t^{n}\right)$, function $f\left(x_{i}, t^{n}\right) \rightarrow f_{i}^{n}$, first time derivative $\left.\frac{\partial f_{i}^{n}}{\partial t} \rightarrow f_{t}\right|_{i} ^{n}$, and second space derivative $\left.\frac{\partial^{2} f_{i}^{n}}{\partial t^{2}} \rightarrow f_{x x}\right|_{i} ^{n}$.

The 2nd Order Central Space approximation of the second derivative is:

$$
\left.f_{x x}\right|_{i} ^{n}=\frac{f_{i+1}^{n}-2 f_{i}^{n}+f_{i-1}^{n}}{\triangle x^{2}}
$$

To obtain second order precision in the time, a key point of the method is to estimate derivatives at half integral time steps. The 2nd Order Central Time approximation of the 
first derivative is given by:

$$
\left.f_{t}\right|_{i} ^{n+\frac{1}{2}}=\frac{f_{i}^{n+1}-f_{i}^{n}}{\triangle t}
$$

The second space derivative is

$$
\left.f_{x x}\right|_{i} ^{n+\frac{1}{2}}=\frac{1}{2}\left(\left.f_{x x}\right|_{i} ^{n+1}+\left.f_{x x}\right|_{i} ^{n}\right) .
$$

The future time level occurs at $n$, the past time level at $n-1$ and the algebraic relationship for the one-dimensional approximation of the Schrödinger equation is:

$$
i\left(\frac{\Psi_{i}^{n}-\Psi_{i}^{n-1}}{\triangle t}\right)=-\frac{1}{4}\left(\frac{\Psi_{i+1}^{n}-2 \Psi_{i}^{n}+\Psi_{i-1}^{n}}{\triangle x^{2}}+\frac{\Psi_{i+1}^{n-1}-2 \Psi_{i}^{n-1}+\Psi_{i-1}^{n-1}}{\triangle x^{2}}\right) .
$$

Then the Crank-Nicholson difference equation is:

$$
-\Psi_{i+1}^{n}+\mathbb{C} \Psi_{i}^{n}-\Psi_{i-1}^{n}=\Psi_{i+1}^{n-1}-\mathbb{C}^{\prime} \Psi_{i}^{n-1}+\Psi_{i-1}^{n-1}
$$

with $\mathbb{C}=(2-i \rho), \mathbb{C}^{\prime}=(2+i \rho)$, and $\rho=\frac{4 \triangle x^{2}}{\Delta t}$.

The algebraic relationship develops a tri-diagonal system represented by the following equation

$$
\widehat{A} \Psi^{n}=\vec{b}
$$

of a set of imax simultaneous linear equations, where imax is the maximum number of grid points , imax - 1 equations come from the interior scheme and one equation comes from the Right Transparent Boundary Condition (at $i=i \max$ ). The left boundary at $i=0$ is still a Dirichlet Boundary Condition and it is set to zero in order to force the function to stay in the domain.

The code presented in this work was based on the design of DTBCs derived by the work of Arnold $\underline{\underline{8} \cdot \underline{9}}$ with the inclusion of modifications that adapt DTBCs to the particular example of the escape problem. The original problem as conceived by Arnold and derived in the dissertation of his student, Earhardt $\underline{10}$, evaluates the transport of a quantum particle that enters one side of a finite domain and exits from the opposite side.

The Right TBC is: 


$$
\Psi_{x}(L, t)=-\sqrt{\frac{2}{\hbar \pi}} e^{-i \frac{\pi}{4}} e^{-i \frac{V_{L}}{\hbar} t} \frac{d}{d t} \int_{0}^{t} \frac{\Psi(L, \tau)}{\sqrt{t-\tau}} e^{+i \frac{V_{L}}{\hbar} t} d \tau .
$$

In contrast with Dirichlet conditions, they are not stagnant in time and the numerical method requires the storage of the past history values at all time levels at the boundaries. The convolution term on the right hand side of the equation (38) appears as a fractional $\left(\frac{1}{2}\right)$ time derivative. Derivation of the DTBCs uses the Crank-Nicholson scheme at a discrete level. The discrete TBCs for the one dimensional Schrödinger equation for $n \geqslant 1$ on the right is at $j=J$

$$
\Psi_{J-1}^{n}-l_{J}^{n} \Psi_{J}^{n}=\sum_{k=1}^{n-1} l_{J}^{(n-k)} \Psi_{J}^{k}-\Psi_{J-1}^{n-1}
$$

with,

$$
\begin{aligned}
l_{j}^{n} & =\left(1-i \frac{R}{2}+\frac{\sigma_{j}}{2}\right) \delta_{n}^{0}+\left(1+i \frac{R}{2}+\frac{\sigma_{j}}{2}\right) \delta_{n}^{1}+\alpha_{j} e^{-i n \varphi_{j}} \frac{P_{n}\left(\mu_{j}\right)-P_{n-2}\left(\mu_{j}\right)}{2 n-1}, \\
R & =\frac{4}{\hbar} \frac{(\triangle x)^{2}}{\triangle t}, \varphi_{j}=\arctan \frac{2 R\left(\sigma_{j}+2\right)}{R^{2}-4 \sigma_{j}-\sigma_{j}^{2}} \\
\mu_{j} & =\frac{R^{2}+4 \sigma_{j}+\sigma_{j}^{2}}{\sqrt{\left(R^{2}+\sigma_{j}^{2}\right)\left(R^{2}+\left[\sigma_{j}+4\right]^{2}\right)}}, \sigma_{j}=2(\triangle x)^{2} V_{j} \\
\alpha_{j} & =\frac{i}{2} \sqrt[4]{\left(R^{2}+\sigma_{j}^{2}\right)\left(R^{2}+\left[\sigma_{j}+4\right]^{2}\right)} e^{i \frac{\varphi_{j}}{2}} .
\end{aligned}
$$

The Right discrete boundary condition is derived at $i=i \max -1$

$$
\begin{array}{r}
-\Psi_{\text {imax }}^{n}+\mathbb{C}_{\text {imax }-1}^{n}-\Psi_{\text {imax }-2}^{n}= \\
\Psi_{\text {imax }}^{n-1}-\mathbb{C}^{\prime} \Psi_{\text {imax }-1}^{n-1}+\Psi_{\text {imax }-2}^{n-1}=b^{n-1}(i \max -1)
\end{array}
$$

where

$$
i=L, \Psi_{\text {imax }}^{n}=\Psi(L, t)
$$

the Right Transparent Boundary Condition, is part of the system of equations. The Discrete Transparent Boundary Condition (38) is:

$$
\begin{array}{r}
\Psi_{\text {imax }-1}^{n+1}-l_{\text {imax }}^{(0)} \Psi_{\text {imax }}^{n+1}= \\
\sum_{k=1}^{n-1} l_{\text {imax }}^{(n-k)} \Psi_{\text {imax }}^{k}-\Psi_{\text {imax }-1}^{n}=b^{n-1}(\text { imax })
\end{array}
$$

Note that $b^{n-1}($ imax $)$ will change at every time level $n$. This is the value at that boundary 
that must be stored by the selected numerical method. For the purpose of illustrating the procedure the first $n=3$ equations are:

$$
\begin{array}{r}
n=1 \\
\Psi_{i \max -1}^{1}-l_{\text {imax }}^{(0)} \Psi_{\text {imax }}^{1}= \\
-\Psi_{\text {imax }-1}^{0}=b^{0}(i \max ) \\
n=2 \\
\Psi_{\text {imax }-1}^{2}-l_{\text {imax }}^{(0)} \Psi_{\text {imax }}^{2}= \\
\Psi_{\text {imax }}^{1} \Psi_{\text {imax }}^{1}=b^{1}(i \max ) \\
\Psi_{\text {imax }-1}^{3}-l_{\text {imax }}^{(0)} \Psi_{\text {imax }}^{3}=3 \\
l_{\text {imax }}^{2} \Psi_{\text {imax }}^{1}+l_{\text {imax }}^{1} \Psi_{\text {imax }}^{2}-\Psi_{\text {imax }-1}^{2}=b^{2}(i \max )
\end{array}
$$

In matrix form,

$$
\left[\begin{array}{cccccc}
\mathbb{C} & -1 & 0 & 0 & 0 & \cdots \\
-1 & \mathbb{C} & -1 & 0 & 0 & \cdots \\
0 & -1 & \mathbb{C} & -1 & 0 & \cdots \\
\vdots & \vdots & \ddots & \ddots & \ddots & \vdots \\
0 & 0 & \cdots & -1 & \mathbb{C} & -1 \\
0 & 0 & 0 & \cdots & -1 & l_{\text {imax }}^{(0)}
\end{array}\right]\left[\begin{array}{c}
\Psi_{2}^{n} \\
\Psi_{3}^{n} \\
\Psi_{4}^{n} \\
\vdots \\
\Psi_{\text {imax-1 }}^{n} \\
\Psi_{\text {imax }}^{n}
\end{array}\right]=
$$

A code called $R T B C$ was written in $C$ which employed the $L U$ Factorization package to solve the complex valued tri-diagonal system of simultaneous linear equations numerically. 


\section{RESULTS.}

Since we use the implicit method called Crank-Nicholson with a degree of approximation through second order that is unconditionally stable, there is no formal restriction on the step sizes, and we expected little difference between the results obtained by both methods. The real and imaginary parts of the wave function are plotted for a set of different values of the time. For simplicity, dimensionless units are used where $m=\hbar=1$. In these units we choose the length of the region to be $L=2$. The set begins with two small values of time and ends with a relatively longer value compared with the value of the natural period. In dimensionless units, $T=\frac{2 \pi}{\omega}=5.092$, for the first ground state with a value of $\omega=1.233$.

\section{A. ANALYTICAL METHOD.}

The exact, time dependent wave function on the semi-infinite domain is obtained by replacing the value of the initial condition in the equation (29) as follows:

$$
\Psi(x, t)=i \frac{\alpha C}{\sqrt{t}} \int_{0}^{L} d x^{\prime}\left(-e^{\frac{i a}{t}\left(x^{\prime}-x\right)^{2}}+e^{\frac{i a}{t}\left(x^{\prime}+x\right)^{2}}\right) \sin \left(k x^{\prime}\right)
$$

After performing the integration using Mathematica Software, the explicit form of the wave function is

$$
\begin{gathered}
\Psi(x, t)= \\
\frac{1}{\sqrt{2}}\left[\left(\left(\frac{1}{4}-\frac{i}{4}\right)(-1)^{\frac{1}{4}} e^{-\frac{i \pi(\pi t+2 a L x)}{2 a L^{2}}}\right)\right. \\
\left(\operatorname{Erfi}\left[\frac{\left(\frac{1}{2}+\frac{i}{2}\right)(-\pi t+a L(L-x))}{\sqrt{a} L \sqrt{t}}\right]\right. \\
-e^{\frac{2 i \pi x}{L}} \operatorname{Erfi}\left[\frac{\left(\frac{1}{2}+\frac{i}{2}\right)(\pi t+a L(L-x))}{\sqrt{a} L \sqrt{t}}\right] \\
-e^{\frac{2 i \pi x}{L}} \operatorname{Erfi}\left[\frac{\left(\frac{1}{2}+\frac{i}{2}\right)(-\pi t+a L(L+x))}{\sqrt{a} L \sqrt{t}}\right] \\
\left.\left.+\operatorname{Erfi}\left[\frac{\left(\frac{1}{2}+\frac{i}{2}\right)(\pi t+a L(L+x))}{\sqrt{a} L \sqrt{t}}\right]\right)\right]
\end{gathered}
$$

where $\operatorname{Erfi}$ is the error function with imaginary argument ${ }^{12}$. 


\section{B. NUMERICAL METHOD.}

A code based on the Crank-Nicholson algorithm was developed to solve the TBC problem. All of the code was written in the $\mathrm{C}$ programming language and the calculations were performed on a LINUX workstation using the GNU compiler. The major effort has gone into adapting the techniques of $T B C s$ because it is complicated by the fact that the boundary condition is non-local in the time, but rather depends on a convolution related to fractional calculus that cannot be treated by standard techniques. The standard algorithm had to be modified to include the non-local, time dependent boundary conditions, which now have to be updated at each time step. To solve the Schrödinger equation on the finite interval, the $L U$ decomposition method was employed. The function libraries were obtained from the open source literature for the GNU compiler.

\section{COMPARISON OF THE METHODS.}

The first set of plots compare the real part of the wave function for both methods at the same time steps $t=0.1, t=0.2$, and $t=1.4$ as shown in figures 2 and 3 .

The second set of plots, figures 4 and 5, compare the imaginary part of the wave function for both methods at the same time steps $t=0.01, t=0.1$, and $t=1.4$.

The comparison between the plots for each method shows an excellent agreement as we expected. This confirms the adequate implementation of the numerical method for a QIVP. Therefore, the basis can be established for a more complex version of the $R T B C$.

\section{CONCLUSIONS.}

In this work we investigated the problem of solving the time dependent Schrodinger equation within a confined region when a constraint is suddenly removed. We accomplished two important goals; the first one is of physical significance. We determined the wave function at any time $t$, and therefore provided all the physical information for the evolution

of the EP within the trap. Therefore, we are able to extract important physical properties of the system such as the mean fraction of mass inside the region, which is called the survival probability, as well as the time dependence of the properties of the particle kinetic energy, potential energy, the total energy, etc.. Since it is assumed that the particle density in the 
system is small, interactions can be ignored (it is a Knudsen Gas ${ }^{11}$ ), and multiplying the survival probability by the total number of particles gives us a quantitative value of mass inside the region.

The second conclusion concerns the numerical significance. We have shown that the discretization developed by Arnold and Ehrhardt for the solution of open boundary problems provides a viable numerical approach for solving the time dependent Schrodinger equation ${ }^{8-10}$ when a constraint is suddenly removed. Although the present work considers a one-dimensional model that is not sufficient to exhibit chaos, the code design forms the essential basis of future research on non-integrable, two-dimensional billiard models where chaos is present. The influence of chaos in these models will be explored by measuring the survival probability of particles in a trap with an open boundary.

The results obtained with the code presented here show an excellent agreement with the analytic approach, offering reliability not only in the result obtained but also in the novel numerical method used. The success in the development of the numerical method opens up a reasonable extension to higher dimensional models. Also, it offers the possibility of understanding the quantum mechanical version of billiard models that experience classical chaotic behavior. In spite of the very low, by normal standards, temperature regime, a classical theoretical model was still adequate for the analysis of the Austin experiment. The quantum regime should also be accessible experimentally at yet lower, but still attainable, temperatures. Experimentalists are beginning to probe this regime.

\section{Acknowledgments}

The authors benefitted from the suggestions of M. Ehrhardt, A. Arnold and K. Yawn, and the support of the division of Technology Resources at Texas Christian University.

1 Oliver Knill,"Sinai billiard", $<$ http://www.dynamical-systems.org/sinai/info.html $>,(2000)$.

2 David M. Harrison,"Bunimovich stadium", $<$ http://www.upscale.utoronto.ca/GeneralInterest /Harrison/Flash/Chaos/Bunimovich/Bunimovich.html>,(2006).

3 Eric W. Weisstein, "Billiards", $<$ http://mathworld.wolfram.com/Billiards.html $>,(2009)$. 
4 Shuiqing $\mathrm{Hu}$ and Arvind Raman,"Chaos in Atomic Force Microscopy," Phys. Rev. Lett. 96, 036107-1-4 (2006).

5 Mason A. Porter and Richard L. Liboff,"Chaos on the Quantum Scale," American Scientist 89, $532-537$ (2001).

6 H. E. Lehtihet and B. N. Miller, "Numerical Study of a Billiard in a Gravitational Field," Physica D 21, 93-104 (1986).

7 V. Milner and J. L. Hanssen and W.C. Campbell and M.G. Raizen, "Optial Billiards for Atoms," Phys. Rev. Lett. 86 1514-1517 (2001).

8 A. Arnold,"Mathematical concepts of open quantum boundary conditions," Trans. Theory Stat. Phys. 30561-584 (2001).

9 M. Ehrhardt and A. Arnold,"Discrete transparent boundary conditions for the Schrödinger equation,"Revista di Matematica della Universita di Parma 657-108 (2001).

10 Matthias Ehrhardt,Discrete Artificial Boundary Conditions(Dissertation, Berlin, 2001).

11 Kerson Huang, "Bose-Einstein condensation of a Knudsen gas," Electronic Resource Cornell University Library, $<$ http://arxiv.org/abs/0708.2234 >,(2007).

12 M. Abromowitz and Irene A. Stegun, Handbook of Mathematical Tables(National Bureau of Standards, Applied Mathematics Series 55, USA, 1985).

13 Joe D. Hoffman, Numerical Methods for Engineers and Scientists(Marcel Dekker, New York, 2001)pp 587-650. 

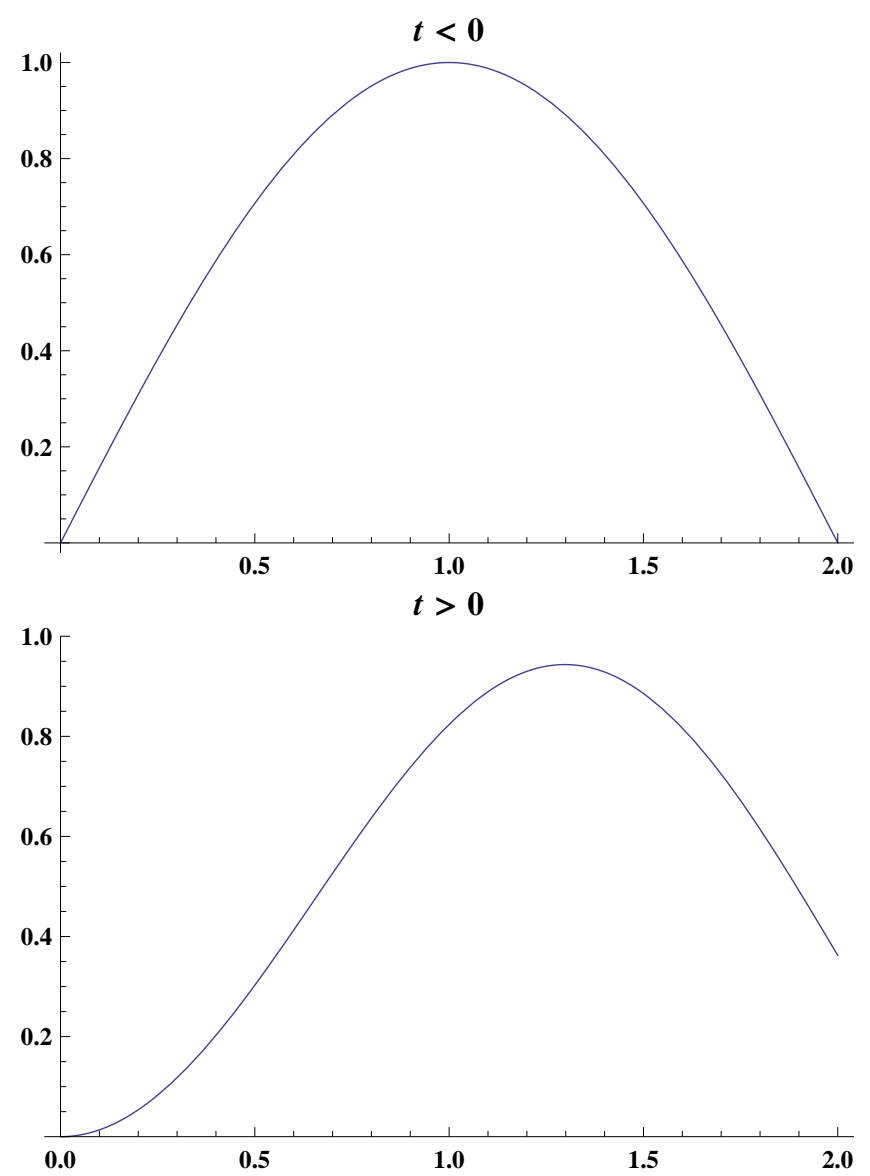

FIG. 1: Top: The EP at time $t<0$, the right boundary is closed. Bottom: The EP at time $t>0$, the right boundary is transparent allowing the wave function to escape.

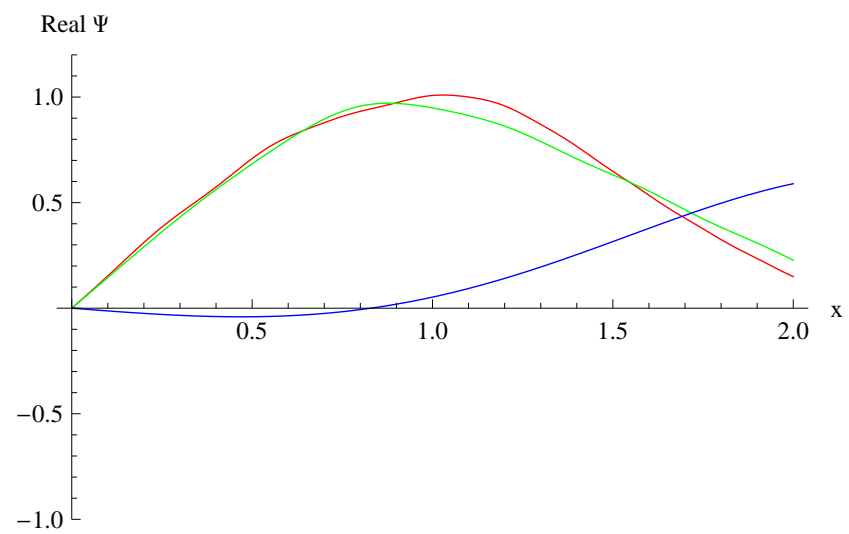

FIG. 2: Real part of the wave function obtained analytically. 


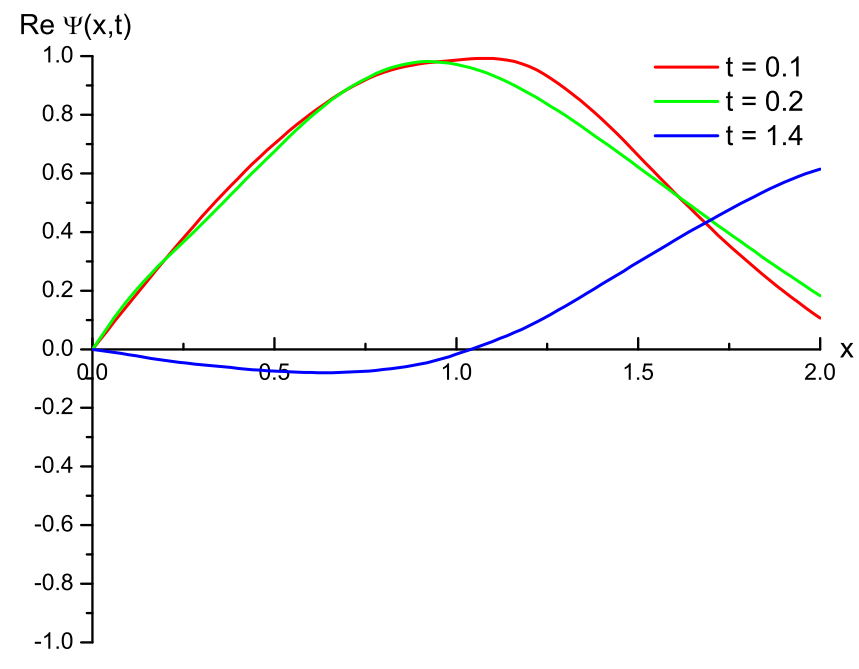

FIG. 3: Real part of the wave function obtained numerically.

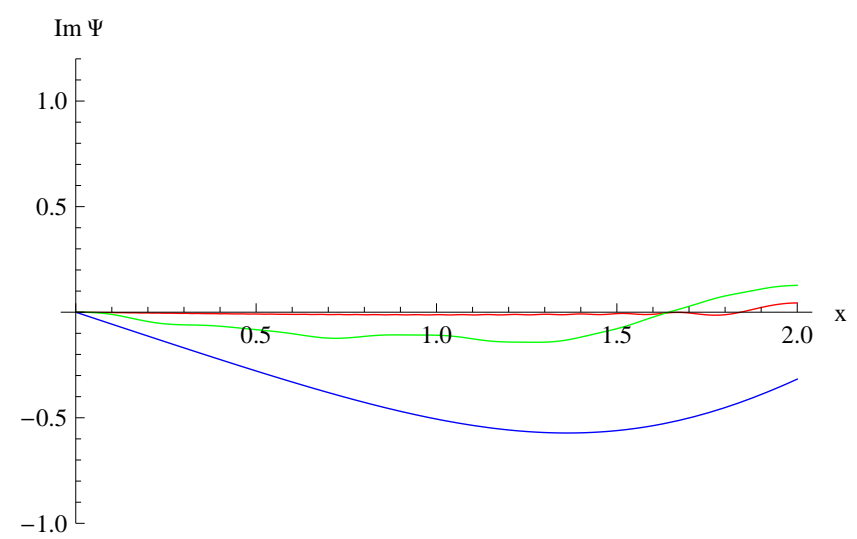

FIG. 4: Imaginary part of the wave function obtained analytically. 


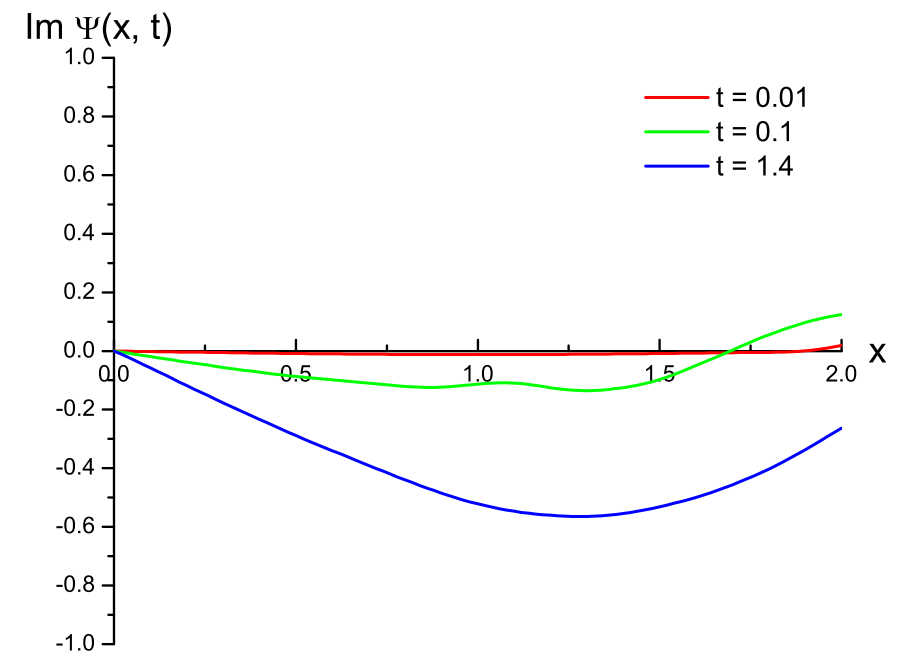

FIG. 5: Imaginary part of the wave function obtained numerically. 